\title{
Antiresonant Hollow Core Fiber with an Octave Spanning Bandwidth for Short Haul Data Communications
}

\author{
J.R. Hayes, Member, OSA, S.R. Sandoghchi, Member, IEEE, Member, OSA T.D. Bradley, Member, \\ OSA, Z. Liu, R. Slavík, Senior Member, IEEE, Member, OSA, M.A. Gouveia, N.V. Wheeler, G. \\ Jasion, Y. Chen, Member, OSA, E. Numkam Fokoua, M.N. Petrovich, Senior Member, IEEE, Senior \\ Member, OSA, D.J. Richardson, Fellow, IEEE, Fellow, OSA and F. Poletti, Member, IEEE
}

\begin{abstract}
We report an effectively single mode tubular antiresonant hollow core fiber with minimum loss of $\sim 25 \mathrm{~dB} / \mathrm{km}$ at $\sim 1200 \mathrm{~nm}$, and an extremely wide low loss transmission window (lower than $30 \mathrm{~dB} / \mathrm{km}$ loss from $1000 \mathrm{~nm}$ to $1400 \mathrm{~nm}$ and $6 \mathrm{~dB}$ bandwidth exceeding $1000 \mathrm{~nm}$ ). Despite the relatively large mode field diameter of $32 \mu \mathrm{m}$, the fiber can be interfaced to SMF28 to produce fully connectorized samples. Exploiting an excellent modal purity arising from large modal differential loss and low intermodal coupling, we demonstrate penalty-free 10G on-off keying data transmission through $100 \mathrm{~m}$ of fiber, at wavelengths of 1065, 1565 and $1963 \mathrm{~nm}$.
\end{abstract}

Index Terms-Fiber optics communications, hollow core optical fibers, low latency, microstructured optical fibers.

\section{INTRODUCTION}

$\mathrm{H}$ OLLOW core fibers based on photonic bandgap guidance (HC-PBGFs) have proven themselves an attractive technology for low latency data transmission [1], with experiments over length scales of practical relevance ranging from a few [2] to a few tens of $\mathrm{km} \mathrm{[3]} \mathrm{so} \mathrm{far} \mathrm{demonstrated.}$ The transmission bandwidth of the single low-loss operating window for HC-PBGFs currently ranges from $\sim 20 \mathrm{~nm}$ for the lowest loss fiber $(1.7 \mathrm{~dB} / \mathrm{km}$ minimum) reported to date [4], to $\sim 200 \mathrm{~nm}$ [2], [5] for fibers with more extended bandwidth but slightly higher loss $(\sim 5 \mathrm{~dB} / \mathrm{km})$. The operating bandwidth is typically centered either on the $\mathrm{C}$-band or at their wavelength of predicted minimum loss, around $2 \mu \mathrm{m}$. While very high data capacity can be achieved in these fibers using dense WDM schemes [6], cheaper ultra-coarse WDM transmission and/or additional capacity would be enabled with an increased bandwidth. A significantly broader bandwidth can be achieved in air-core antiresonant fibers (ARFs) where guidance is based on antiresonance from thin glass membranes

This work was partly funded by the UK EPSRC through grant EP/I061196X. FP and DJR acknowledge support from the Royal Society.

All authors are with the Optoelectronics Research Centre, University of Southampton, Southampton, SO17 1BJ, UK (e-mail: jrh@orc.soton.ac.uk; srs1g12@soton.ac.uk; T.Bradley@soton.ac.uk; z.liu@soton.ac.uk; r.slavik@soton.ac.uk; mag2g12@soton.ac.uk; nvw1v10@orc.soton.ac.uk; G.Jasion@soton.ac.uk; yc1m12@soton.ac.uk; ernf1g10@orc.soton.ac.uk; mnp@orc.soton.ac.uk; djr@orc.soton.ac.uk; frap@orc.soton.ac.uk). surrounding the hollow core, and the cladding consists of either a Kagome-type lattice [7], or a single ring of touching [8] or non-touching tubes [9], [10]. However, the vast majority of ARFs reported to date, including all those with a Kagome lattice and with touching tubes, exhibit a structured transmission spectrum, with multiple loss peaks arising from resonant coupling between air and glass modes located at nodes in the cladding. These resonances are difficult to control and give rise both to higher loss (typically $\sim 50-300 \mathrm{~dB} / \mathrm{km}$ ) and poorly reproducible modal properties. Consequently, such fibers are unlikely candidates for data transmission over hollow core fiber. We note that to the best of our knowledge, no data transmission experiments have yet been reported in any form of air-core antiresonant fiber).

In this work we demonstrate for the first time that this emerging fiber technology has the potential to make an impact on short reach data transmission. We present a non-contacting tubular fiber, comprising a single ring of 7 tubes around a hollow core (Fig.1a), with the widest sub-100 dB/km transmission window $(>1000 \mathrm{~nm}$ or $>1$ octave centered at $1380 \mathrm{~nm})$ ever reported for any air-guiding fiber and a minimum loss of $\sim 25 \mathrm{~dB} / \mathrm{km}$. Due to its high differential modal loss and low intermodal coupling, an excellent modal purity ( $>50 \mathrm{~dB}$ suppression of high order modes) is achieved after propagation lengths of just a few $10 \mathrm{~s}$ of meters. This, combined with a spectrally smooth loss curve, allows lowlatency data transmission over an unprecedentedly broad spectral range. To demonstrate this, we report penalty free $10 \mathrm{Gbit} / \mathrm{s}$ data transmission at wavelengths of $1065 \mathrm{~nm}, 1556$ $\mathrm{nm}$ and $1963 \mathrm{~nm}$ in the same $100 \mathrm{~m}$ long fiber [11].

\section{FIBER FABRICATION AND CHARACTERIZATION}

The fibers in this work were produced using a modified two stage stack-and-draw process. We chose to stack a preform with 7 tubes since modelling indicated that this would produce a good trade-off between low straight loss, low bend loss, and large differential mode loss (i.e. good modal purity after a sufficiently long propagation length). The manufacture of uniform capillaries to form the cladding tubes $(4.09 \pm 0.02$ $\mathrm{mm}$ diameter and $0.24 \mathrm{~mm}$ thick walls) was a key fabrication step, since, as we will show later in this work, the loss of 
tubular ARFs and their high order mode extinction ratio depend critically on the regularity of their structure. Dimensional differences amongst the final tubes in the fiber cause additional light leakage between the gaps and decrease the bandwidth due to broadening of the resonance peaks. Since during the second draw stage tube pressurization was necessary to increase the initial aspect ratio (inner to outer diameter ratio) of the stacked capillaries from $\sim 0.94$ to the final $\sim 0.98$, any initial difference between the capillaries would tend to be magnified in the final fiber by this process. The fiber draw parameters were slightly tuned during the draw to produce fibers centered at different wavelengths and with core diameters ranging between $\sim 38 \mu \mathrm{m}$ and $\sim 45 \mu \mathrm{m}$. The target fiber outer diameter was set to either $180 \mu \mathrm{m}$ or $200 \mu \mathrm{m}$. The yield from one preform was $\sim 2.1 \mathrm{~km}$, but we envisage it could be straightforwardly scaled up to $>50 \mathrm{~km}$ per meter of preform [12].

Fig. 1 shows a scanning electron micrograph of one of the fabricated fibers (core diameter of $40.2 \mu \mathrm{m}$, optical properties shown in Fig.3).

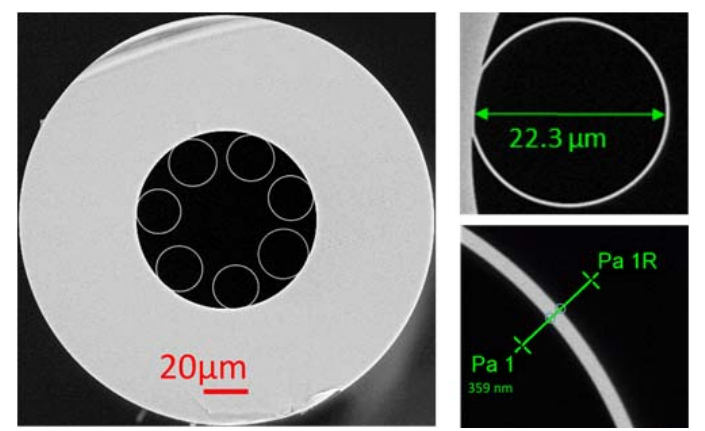

Fig. 1. Electron micrographs of the full fiber cross section and details of the cladding of Fiber $\mathrm{C}$ having core diameter $40.2 \mu \mathrm{m}$.

Our aim in this work was to demonstrate the potential of this fiber type in a fully fiberized data transmission experiment, for which connectivity to standard single mode fiber (SMF) is required. Accordingly, we developed mode matched splice recipes to allow low-loss interconnection to SMF employing a suitable intermediate buffer fiber (see below). Loss measurements were performed via a cutback method employing a supercontinuum source, two optical spectrum analyzers (400-1750 nm and 1200-2400 nm, $2 \mathrm{~nm}$ resolution) to cover the full transmission bandwidth of the fiber, and the input end mode-matched and spliced to SMF. We characterized the loss of three different fibers (A, B and C) with slightly different core size and glass membrane thickness, leading to shifted transmission bands. Fig. 2 shows the loss of one fiber (Fiber A) having a core diameter of 38 $\mu \mathrm{m}$, cutback from $145 \mathrm{~m}$ to $4 \mathrm{~m}$. The loss oscillations visible at $\sim 870,1180$ and $1400 \mathrm{~nm}$ are believed to be due to some modal beating occurring in the short fiber length that remained after the cut-off $(4 \mathrm{~m})$, since they were present in both short and long length transmission spectra. Despite this, the minimum measured loss was $<20 \mathrm{~dB} / \mathrm{km}$, and the loss was $<100 \mathrm{~dB} / \mathrm{km}$ over the wavelength range $785 \mathrm{~nm}$ to $1705 \mathrm{~nm}$. A similar fiber with slightly thicker tubes (Fiber B, not shown) had a minimum loss of $\sim 25 \mathrm{~dB} / \mathrm{km}$ and $<120 \mathrm{~dB} / \mathrm{km}$ for all wavelengths between $900 \mathrm{~nm}$ and $2050 \mathrm{~nm}$.

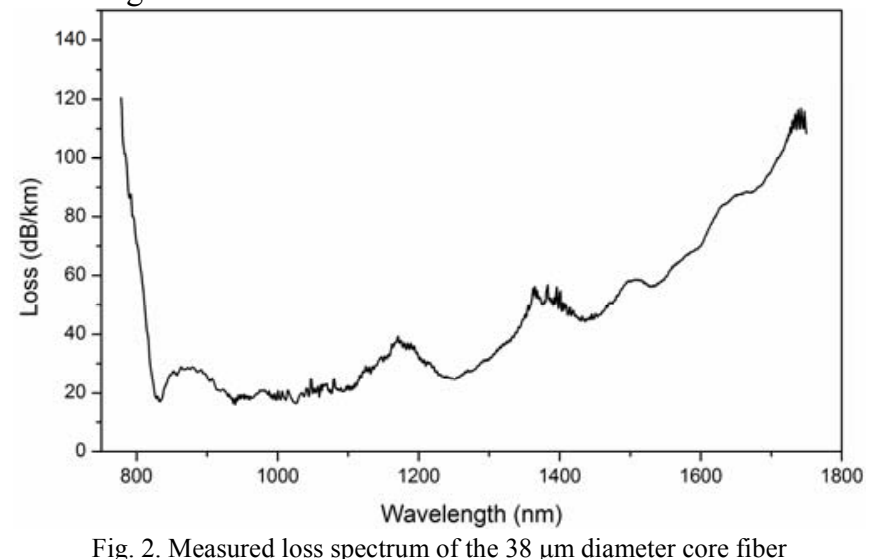

Figure 3 shows the loss spectrum of a third fiber (Fiber C), 100 meters long, that we have selected for more detailed analysis and for our data transmission experiment. It has a core diameter of $40.2 \mu \mathrm{m}$, an average tube diameter of 22.6 $\mu \mathrm{m}$ (with a standard deviation of $0.9 \mu \mathrm{m}$ ) and an average membrane thickness of $\sim 360 \mathrm{~nm}$ (Fig.1). Its cutback loss is compared in Fig.3 to the lowest reported loss HC-PBGF [4] and Kagome ARF [7], and to a wide bandwidth HC-PBGF [5], to highlight the exceptionally wide $(>1000 \mathrm{~nm})$ air-guiding bandwidth of this fiber. Unlike other ARF types reported in the literature the loss is spectrally smooth and extremely flat over a $400 \mathrm{~nm}$ wide region between 1000 and $1400 \mathrm{~nm}$ where it is below $30 \mathrm{~dB} / \mathrm{km}$. Its minimum loss is $26 \mathrm{~dB} / \mathrm{km}$ at 1200 $\mathrm{nm}$. Note that besides this fundamental window of operation, the fiber also guides in high order windows at shorter wavelengths, which we haven't fully characterized yet. Exploiting the lower loss predicted from theory for the second antiresonant window, a tubular lattice ARF with 8 tubes and record loss of $7.7 \mathrm{~dB} / \mathrm{km}$ at $750 \mathrm{~nm}$ was recently reported [13].

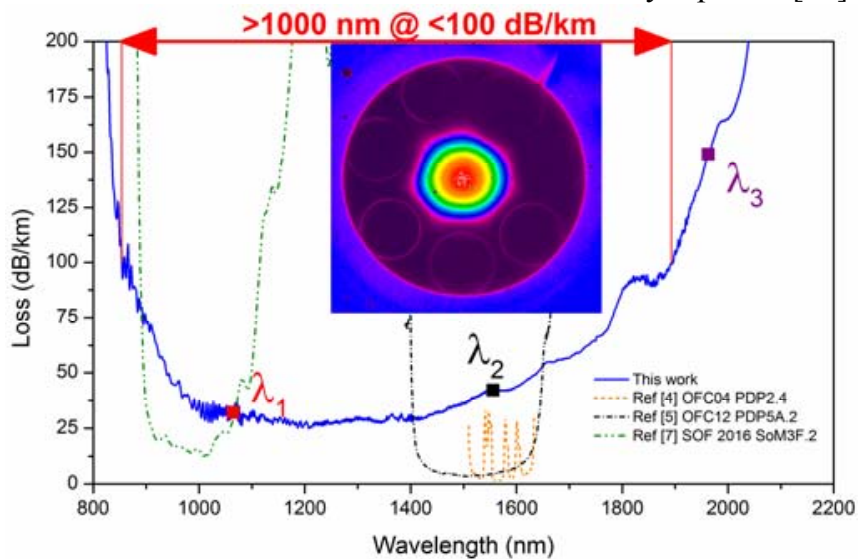

Fig.3. Measured loss spectrum (blue curve) compared with state-of-the-art hollow core PBGFs (orange/black) and Kagome ARF (green) highlighting the exceptional bandwidth and spectral flatness of the present fiber. Inset: measured mode image after 5 meters.

Fig.4 shows other measured and simulated optical properties of the fiber. An advantageous characteristic for data transmission is the very low group velocity dispersion of $<2.5$ $\mathrm{ps} / \mathrm{nm} / \mathrm{km}$ across the whole transmission window (as expected 
for a mode that travels mainly in air and with little overlap with the glass). The fiber also has a large mode field diameter (MFD), which is almost constant at $\sim 32 \mu \mathrm{m} \pm 5 \%$ across the full octave, and which, in contrast to conventional, simple step index, single mode fiber, decreases with wavelength We note it continues to decrease for wavelengths longer than the most anti-resonant wavelength (which corresponds to twice the frequency of the first high loss resonance in this window). We attribute this to the radially inwards movement of the first null in the electric field as the electric field in the glass layer broadens with increasing wavelength. This is qualitatively similar to the response of the fundamental mode of a step index slab waveguide.

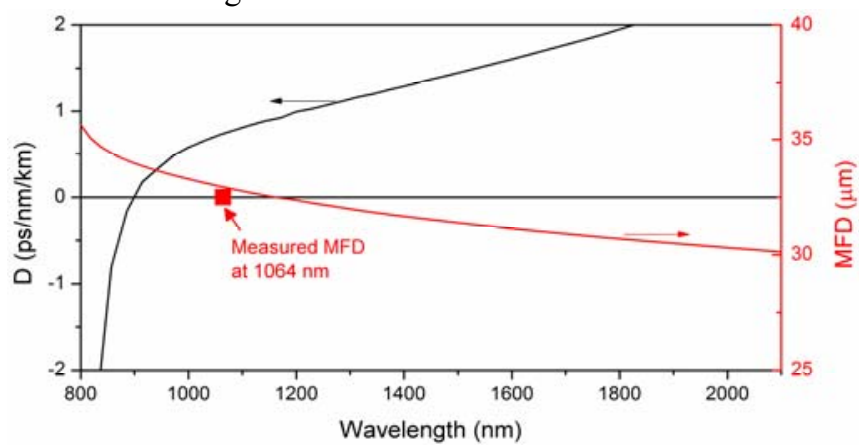

Fig. 4. Simulated dispersion (D) and mode field diameter (MFD) of fiber C, with superimposed measurement at $1064 \mathrm{~nm}$.

Using a calibrated FEM modelling tool [14], we investigated the effect of structural non-uniformities in the fiber, and the potential for further improvement in loss and higher order mode (HOM) extinction. Modelling the actual structure by taking into account small non-uniformities (e.g. in the inter-tube gaps), was found to yield excellent agreement with the experimental data, as shown in Fig.5 (red solid curve). This confirms that, as mentioned above, the dominant loss mechanism in these fibers is leakage loss. By contrast, as a result of the antiresonance operation which expels light from the membranes and produces a very small fraction of optical power in the glass ( 1 to $8 \times 10^{-5}$ across the full bandwidth), surface scattering loss, which is prevalent in HC-PBGFs, has only a negligible contribution $(\sim 0.1 \mathrm{~dB} / \mathrm{km}$ for this fiber). Simulations also indicate that simply by improving the structure and achieving perfect regularity a loss reduction down to $10 \mathrm{~dB} / \mathrm{km}$ could be achieved for this very same design (black dashed curve), with lower losses in the few $\mathrm{dB} / \mathrm{km}$ possible for similar but more optimized designs [13].

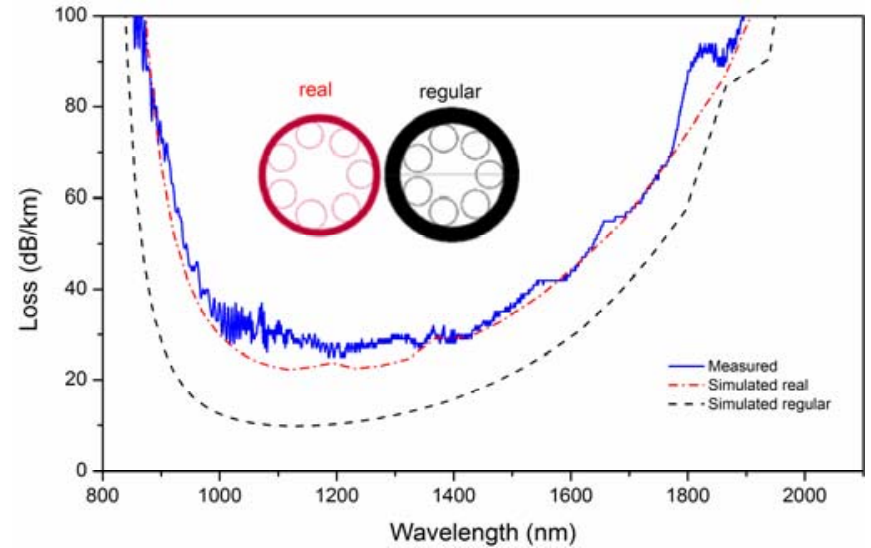

Fig. 5 Measured (blue) and simulated (red) loss of the fiber in Fig.1, and simulated loss of a similar fiber with regular structure (black dash).

The simulated differential mode loss for the real, slightly asymmetric Fiber $\mathrm{C}$, indicates that the high order mode (HOM) with the lowest loss $\left(\mathrm{LP}_{11}\right)$ is over 10 times lossier than the fundamental $\mathrm{LP}_{01}$-like mode (i.e. it has a minimum loss of $\sim 250 \mathrm{~dB} / \mathrm{km}$ ), and the loss of all other high-order modes is over 100 times greater $(>2.5 \mathrm{~dB} / \mathrm{m}$ ), see Figure 6 (red symbols). This is a result of resonant coupling between coreguided modes and air modes guided in the cladding tubes, and is a well understood effect for this type of fiber $[10,11]$. If irregularities in the structure are neglected (black symbols), and even more so if the perfectly symmetric tubes are further enlarged by $10 \%$ (blue symbols), an improved phase-matching is achieved, since the effective indices of the core modes decrease and those of the tube modes increase. Simulations show that as a result of this, the present structure with tubes of identical diameter would produce a larger mode loss ratio (x60) between $\mathrm{LP}_{01}$ and the lowest loss HOM. Increasing the tube diameters by only $10 \%$ would produce a further improvement in mode loss ratio, with high order mode loss ratios of $>100$ in principle achievable. The improvement in differential loss is due to increased out coupling of the HOMs because of the improved similarity between the effective indices of the HOMs of the core and the air guided modes of the cladding tubes.

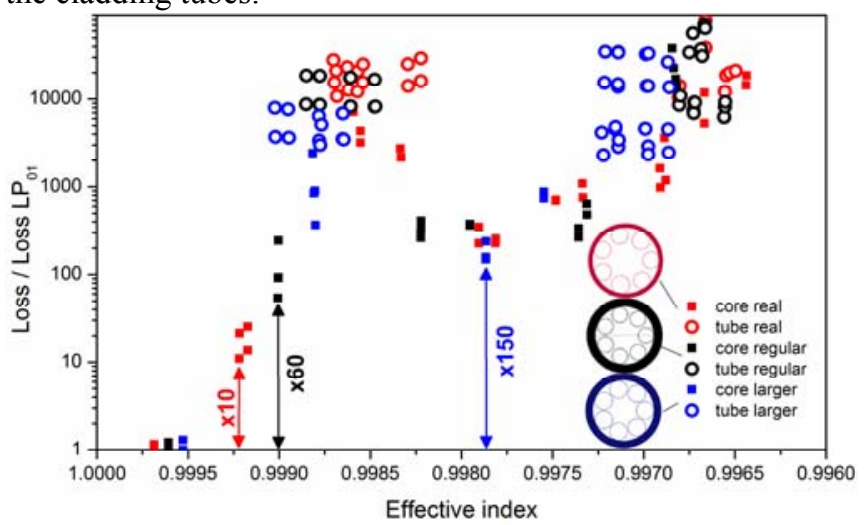

Fig. 6 Simulated loss at $1200 \mathrm{~nm}$, normalized to that of the lowest loss fundamental mode, of the first 50 core modes (squares) and air guided modes of the cladding tubes (hollow circles), as the structure is changed from the real fiber shown in Fig. 1 (red) to a regular structure (black) to one having larger tubes (blue). 
The already fairly large mode loss ratio of Fiber $\mathrm{C}(10 \times$, as compared to $2-3 \times$ of HC-PBGFs [14] but less than $\sim 1000 \times$ calculated for an optimized 6 tube ARF [10]), combined with a very low intermodal coupling due to the small overlap of the field with the glass surfaces (the mechanism for intermodal coupling is akin to that of the scattering loss), together with suitable core mode excitation described below, makes this fiber strongly single moded after just a few 10 s of meters. This prediction is confirmed by the S2 measurements performed over $3 \mathrm{~m}$ and $100 \mathrm{~m}$ long fibre samples and shown in Fig.7. They show that while a small LP11 fractional component (at $\sim-20 \mathrm{~dB}$ level) can be observed after $3 \mathrm{~m}$ meters, no LP11 (or indeed any other mode) component can be observed above the noise floor of the measurement with the $100 \mathrm{~m}$ long fiber sample. The measurements allow us to conclude that the modal purity (high order mode suppression) is better than 50 $\mathrm{dB}$. This is a conservative number obtained in the worst case scenario where intermodal coupling broadens the LP11 peak at $1.7 \mathrm{ps} / \mathrm{m}$ significantly, making its power fall below the measurement floor. Without such consideration, the LP11 mode energy would be all contained in a narrow peak at 1.7 ps/m (Fig. 7), resulting in mode purity better than $75 \mathrm{~dB}$ (limited by the measurement sensitivity, shown at $-80 \mathrm{~dB}$ in Fig. 7).. It confirms that the combination of suitable excitation, intrinsic HOM loss discrimination of the fiber and fiber length does indeed lead to the expected result and verified that distributed coupling to a HOM noted elsewhere [10] should not be a problem in our transmission experiment. This is, to the best of our knowledge, the highest ever experimental demonstration of modal purity reported in a hollow core fiber link to date

In preparation for the transmission experiment, and despite the large MFD mismatch with SMF, we have been able to produce a fully connectorized SMF28 to hollow core to SMF28 fiber link with a total overall connectorization loss of $\sim 2.1 \mathrm{~dB}$ (as of yet not fully optimized) through the use of suitable intermediate buffer fibers with intermediate MFD of the fundamental mode. The S2 measurements and cutback loss shown in Figs. 3 and 5, were performed with the same mode field matching splice assembly on the input side. For the transmission experiments a second splice assembly was joined to the output end of the link.

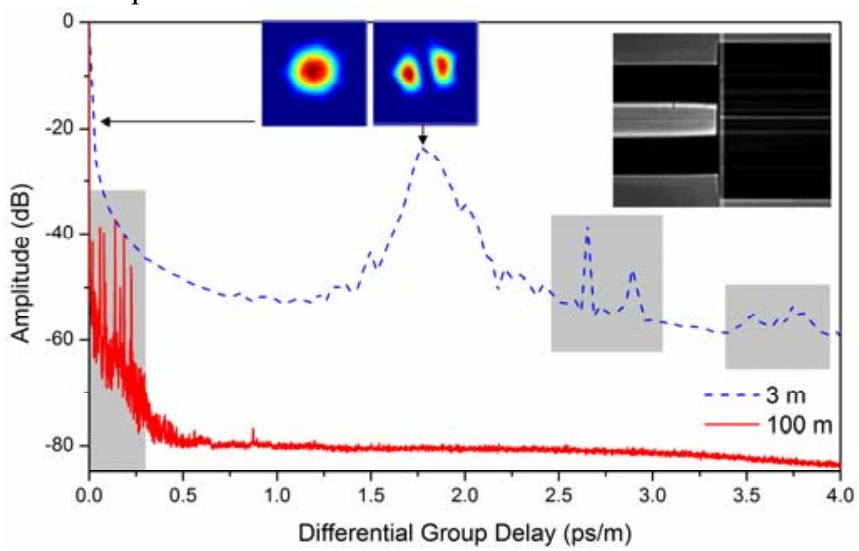

Fig.7. $\mathrm{S}^{2}$ measurement of the fiber with a spliced launch (inset: splice image), indicating some residual high order mode content after $3 \mathrm{~m}$ (blue curve) and a high modal purity obtained after $100 \mathrm{~m}$ (red curve). The grayed area shows known measurement artifacts associated with the detailed measurement set up.

\section{DATA TRANSMISSION EXPERIMENT}

To test the ability of the fiber to transmit data across such a very wide wavelength range we measured the data transmission performance through $100 \mathrm{~m}$ length of fiber at three widely different wavelengths: towards the short wavelength transmission edge at $1065 \mathrm{~nm}$, in the middle of the transmission band at $1565 \mathrm{~nm}$, and at the long wavelength transmission edge at $1963 \mathrm{~nm}\left(\lambda_{1-3}\right.$ in Fig.3). At the three wavelengths of interest the total span loss was 4.9, 6.3 and $18.5 \mathrm{~dB}$, respectively. An on-off keying data rate of $10 \mathrm{Gbit} / \mathrm{s}$ (with a $10^{31}-1$ pseudorandom bit sequence) was used in each instance. Due to the extreme wavelength range covered it was necessary to use different combinations of lasers, modulators and detectors to make the measurements. In each instance the signals were passed through a polarization controller and launched into the fiber under test. At the receiver side the signal was amplified and analyzed with a bit error ratio (BER) tester. To compensate for the higher fiber loss at $1963 \mathrm{~nm}$ a Thulium-doped fiber amplifier was used. In all cases the transmission was essentially BER power penalty-free, regardless of polarization state at the fibre input (that was changed using the polarization controller) confirming the excellent transmission characteristics of the fiber at these widely disparate wavelengths. Fig. 8 a shows the spectral characteristics and eye diagrams for each wavelength after 100 $\mathrm{m}$ transmission and fig. $8 \mathrm{~b}$ the BER. Whilst we have only tested at 3 discrete wavelengths we fully expect the fiber to transmit data equally well across its entire low loss transmission window.
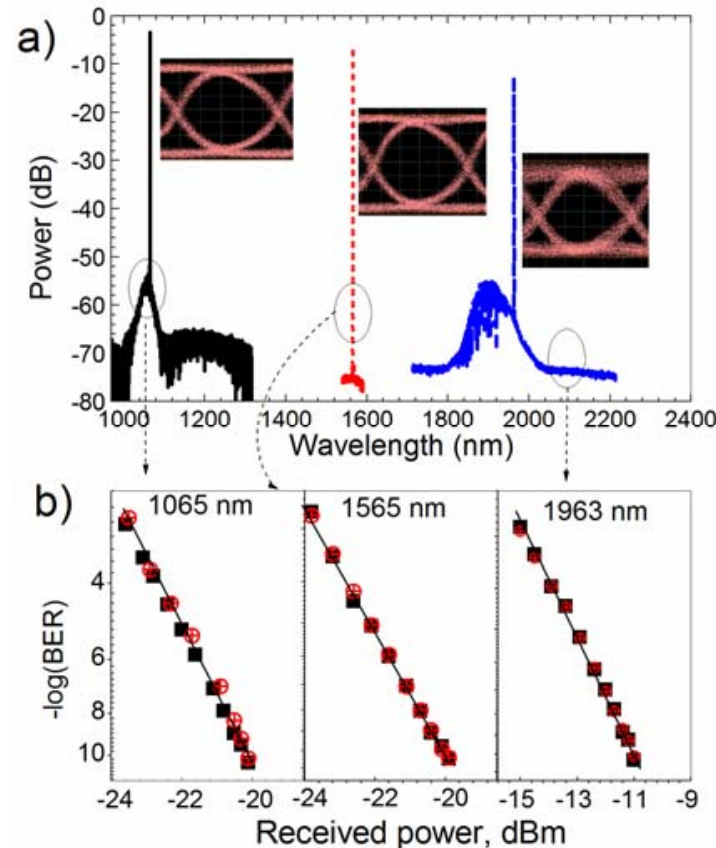

Fig. 8. Data transmission at $10 \mathrm{Gbit} / \mathrm{s}$. a) Spectral characteristics $(0.1 \mathrm{~nm}$ resolution): $1065 \mathrm{~nm}$ (solid black), $1565 \mathrm{~nm}$ (dash red), and $1963 \mathrm{~nm}$ (short dash blue) wavelengths. Eye diagrams after the transmission through 100-m long ARF sample are shown as insets. B) BER curves: back-to-back (black squares) and after transmission (red circles) showing no power penalty. 


\section{CONCLUSIONS}

We have reported the first hollow core anti-resonant fiber capable of data transmission at wavelengths separated by nearly one octave. The present fiber has shown penalty-free data transmission through $100 \mathrm{~m}$ at 1065,1565 and $1963 \mathrm{~nm}$ wavelengths, but future versions could simultaneously cover the perhaps even more attractive wavelengths of 850,1300 and $1550 \mathrm{~nm}$, where the fiber's low latency, dispersion and nonlinearity would be extremely attractive features for short reach communications. Despite its large MFD of $\sim 30 \mu \mathrm{m}$, and in conjunction with the spliced input, after $100 \mathrm{~m}$ the fiber link was experimentally shown to have the purest single mode transmission reported to date over a hollow core fiber. Fully spliced connections with SMF28 were achieved with modest additional loss $(\sim 1 \mathrm{~dB}$ average per connection, with further reduction anticipated). Loss reduction from the reported 25 $\mathrm{dB} / \mathrm{km}$ down to $\sim 10 \mathrm{~dB} / \mathrm{km}$ level or below is possible through realistic improvements of the fiber structure, as demonstrated in recent work [13], making km-scale spans possible, e.g. for intra data center applications. Finally, simulations indicate that through the inclusion of more complicated tubular structures (specifically inner nested tubes), the loss of these fibers is predicted to drop by two further orders of magnitude or more [16], making these antiresonant hollow core fibers one of the most promising novel fiber technologies currently under investigation.

\section{ACKNOWLEDGMENT}

The data for this paper can be found at DOI: $10.5258 /$ SOTON/397853.

\section{REFERENCES}

[1] F. Poletti, N. V. Wheeler, M. N. Petrovich, N. Baddela, E. N. Fokoua J. R. Hayes, D. R. Gray, and D. J. Richardson, "Towards high-capacity fibre-optic communications at the speed of light in vacuum," Nature Photonics, vol. 7, no. 4. pp. 279-284, 2013.

[2] Y. Chen, Z. Liu, S. R. Sandoghchi, G. T. Jasion, T. D. Bradley, E. N Fokoua, J. R. Hayes, N. V Wheeler, D. R. Gray, B. J. Mangan, R. Slavík, F. Poletti, M. N. Petrovich, and D. J. Richardson, "Multi kilometer Long, Longitudinally Uniform Hollow Core Photonic Bandgap Fibers for Broadband Low Latency Data Transmission," J. Light. Technol., vol. 34, no. 1, pp. 104-113, 2016.

[3] M. Kuschnerov, V. A. J. M. Sleiffer, Y. Chen, E. De Man, Y. Chen, S. R. Sandoghchi, G. T. Jasion, T. Bradley, E. N. Fokoua, R. Hayes, N. V Wheeler, D. R. Gray, R. Slavik, Y. Jung, L. Nicholas, B. J. Mangan, F. Poletti, M. N. Petrovich, and D. J. Richardson, "Data Transmission through up to $74.8 \mathrm{~km}$ of Hollow-Core Fiber with Coherent and DirectDetect Transceivers," in ECOC, 2015, paper. Th1.2.4.
[4] J. Mangan, L. Farr, A. Langford, P. J. Roberts, D. P. Williams, F. Couny, M. Lawman, M. Mason, S. Coupland, R. Flea, and H. Sabert, "Low loss $(1.7 \mathrm{~dB} / \mathrm{km})$ hollow core photonic bandgap fiber," in OFC 2004, paper PDP24.

[5] N. V Wheeler, M. N. Petrovich, R. Slavík, N. Baddela, E. Numkam, J. R. Hayes, D. R. Gray, F. Poletti, and D. J. Richardson, "Widebandwidth, low-loss, 19-cell hollow core photonic band gap fiber and its potential for low latency data transmission," in OFC 2012, paper PDP5A.2.

[6] V. A. J. M. Sleiffer, S. Member, Y. Jung, N. K. Baddela, J. Surof, M. Kuschnerov, V. Veljanovski, J. R. Hayes, N. V Wheeler, E. R. N. Fokoua, J. P. Wooler, D. R. Gray, N. H. Wong, S. Member, F. R. Parmigiani, S. Alam, M. N. Petrovich, S. Member, F. Poletti, D. J. Richardson, and H. De Waardt, "High Capacity Mode-Division Multiplexed Optical Transmission in a Novel 37-cell Hollow-Core Photonic Bandgap Fiber," J. Light. Technol., vol. 32, no. 4, pp. 854 863, 2014.

[7] N.V. Wheeler, T.D. Bradley. J.R. Hayes. M.A. Gouveia, Y. Chen, S.R. Sandoghchi, F. Poletti, M.N. Petrovich and D.J. Richardson. "Low Loss Kagome Fiber in the $1 \mu \mathrm{m}$ Wavelength Region," in Specialty Optical Fibres at the OSA Advanced Photonics Congress 2016, paper SoM3F.2.

[8] F. Yu, W. J. Wadsworth, and J. C. Knight, "Low loss silica hollow core fibers for 3-4 $\mu \mathrm{m}$ spectral region," Opt. Express, vol. 20, no. 10, pp. 11153-11158, 2012.

[9] A. N. Kolyadin, A. F. Kosolapov, A. D. Pryamikov, A. S. Biriukov, V. G. Plotnichenko, and E. M. Dianov, "Light transmission in negative curvature hollow core fiber in extremely high material loss region.," Opt. Express, vol. 21, no. 8, pp. 9514-9, 2013.

[10] P. Uebel, M. C. Gunendi, M. H. Frosz, G. Ahmed, N. N. Edavalath, J.M. Menard, and P. S. J. Russell, "Broadband robustly single-mode hollow-core PCF by resonant filtering of higher-order modes," Opt. Lett., vol. 41, no. 9, pp. 1961-1964, 2016.

[11] J. R. Hayes, S. R. Sandoghchi, T. D. Bradley, Z. Liu, R. Slavík, M. A. Gouveia, N. V Wheeler, G. Jasion, Y. Chen, E. N. Fokoua, M. N. Petrovich, D. J. Richardson, and F. Poletti, "Antiresonant Hollow Core Fiber with Octave Spanning Bandwidth for Short Haul Data Communications," in OFC, 2016, paper Th5A.3.

[12] G. T. Jasion, E. Numkam Fokoua, J. S. Shrimpton, D. J. Richardson and F. Poletti, "Studying the limits of production rate and yield for the volume manufacturing of hollow core photonic band gap fibers,". Opt. Express, Vol. 23, no 25, pp. 32179 -32190, 2015.

[13] B. Debord, A. Amsanpally, M. Chafer, A. Baz, L. Vincetti, J. M. Blondy, F. Gérôme, and F. Benabid, "7.7 dB/ km losses in inhibited coupling hollow-core photonic crystal fibers," in CLEO, 2016, paper JTH4C.8.

[14] E. Numkam Fokoua, S. R. Sandoghchi, Y. Chen, G. T. Jasion, N. V. Wheeler, N. K. Baddela, J. R. Hayes, M. N. Petrovich, D. J. Richardson, and F. Poletti, "Accurate modelling of fabricated hollowcore photonic bandgap fibers," Opt. Express 23, $23117-23132$ (2015).

[15] J. W. Nicholson, A. D. Yablon, J. M. Fini and M. D. Mermelstein, "Measuring the Modal Content of Large-Mode-Area Fibers," in IEEE $J$. Sel. Top. Quantum Electron., vol. 15, no. 1, pp. 61-70, 2009.

[16] Francesco Poletti, "Nested antiresonant nodeless hollow core fiber," Opt. Express, Vol. 22, pp. 23807-23828, 2014. 